\title{
Axioms and Variational Problems in Surface Parameterization
}

\author{
Ulrich Clarenz $^{\mathrm{a}, 1}$ Nathan Litke ${ }^{\mathrm{b}, 2}$ Martin Rumpf ${ }^{\mathrm{a}, 3}$ \\ ${ }^{a}$ Duisburg-Essen University, Lotharstrasse 63-65, 47048 Duisburg, Germany \\ ${ }^{\mathrm{b}}$ Caltech, Pasadena, CA 91125, USA
}

\begin{abstract}
For a surface patch on a smooth, two-dimensional surface in $\mathbb{R}^{3}$, low-distortion parameterizations are described in terms of minimizers of suitable energy functionals. Appropriate distortion measures are derived from principles of rational mechanics, closely related to the theory of non-linear elasticity. The parameterization can be optimized with respect to the varying importance of conformality, length preservation and area preservation. A finite element discretization is introduced and a constrained Newton method is used to minimize a corresponding discrete energy. Results of the new approach are compared with other recent parameterization methods.
\end{abstract}

Key words: Surface Parameterization, Non-linear Elasticity, Variational Methods, Finite Element Methods

\section{Introduction}

The aim of this paper is twofold. At first, we present a new parameterization method with a mathematically rigorous variational foundation derived from first principles. It casts the parameterization problem in the language of rational mechanics and provides built-in parameters for the control of angle, area and length distortion. We provide existence proofs for minimizers of the resulting parameterization energies. Secondly, we derive a finite element discretization from the continuous model in a straightforward way. The associated discrete non-linear energy minimization problem can be solved efficiently with a constrained Newton method with step size control. Our overall approach is closely related to the theory of non-linear elasticity

1 E-mail: clarenz@math.uni-duisburg.de.

2 E-mail: njlitke@cs.caltech.edu.

3 Corresponding author. E-mail: rumpf@math.uni-duisburg.de. 
and polyconvex functionals. Throughout, we we will restrict ourselves to surface patches of disc type.

The most desirable property to achieve in a parameterization is isometry. This implies intuitively that all of the properties of the surface are represented in the corresponding parameter domain. Strictly speaking, a map between two surfaces is an isometry if their first fundamental forms coincide. It is well known that isometric parameterizations exist only if the surface itself is locally flat, i.e. developable. A broad variety of algorithms have been proposed to construct such parameterizations for embedded triangle meshes (for a recent survey see the comprehensive overview by Floater and Hormann [1]). Generically these algorithms are distinguished by the way they measure distortion. Examples include linear and non-linear measures of area, length, and angle change. Except for the case of harmonic and conformal maps [2-5], little in the way of theory is available to guide the choice of distortion measures and appropriate numerical methods to minimize them effectively. Consequently it is often unclear whether unique solutions exist and what assertions can be made about the resulting parameterizations. If we cannot preserve all quantities, one may ask for certain quantities which may be preserved by a parameterization, e.g., length, angles, and local area.

The question of an angle preserving, i.e., conformal map has a long history. The question of whether all surfaces can be parameterized conformally was asked by Gauß, who was able to solve this problem for real analytic metrics. An approach for less regular, namely $C^{1, \alpha}$-surfaces is due to Lichtenstein [6]. In this setting, it is possible to parameterize the surface globally in a conformal way using the uniformization theorem. The idea of a proof via direct methods in the calculus of variations dates back to Morrey [7], although his reasoning contained errors and Jost gave a rectified proof [8]. Sauvigny introduced a method of continuity for conformal parameterizations of surfaces [9]. Recently, Hildebrandt and von der Mosel [10] presented a new approach for the conformal parameterization of disctype surfaces which does not make use of the uniformization theorem. For more details concerning the history and literature of conformal mappings we refer the reader to [11, pp. 49] and [8]. Due to the fact that we intend to process triangulated surfaces, all of the above approaches are improper for numerical purposes. Only Morrey's and Jost's method seems (at a first glance) to be numerically useful. But computing a first variation of their functional to be minimized requires higher regularity of the surface than $C^{0,1}$.

On the other hand, from a practical point of view it does not make sense to restrict ourselves to conformal parameterizations. One undesirable property of conformal maps appears in the form of large area distortion effects. A related idea is to provide a parameterization that perfectly preserves area. The theoretical problem of finding such maps has been studied, although not as deeply and detailed as the problem of conformality $[5,6,8]$. Clearly there are strong relationships to the theory of Monge-Ampère equations and mass transfer problems [12]. To our knowl- 

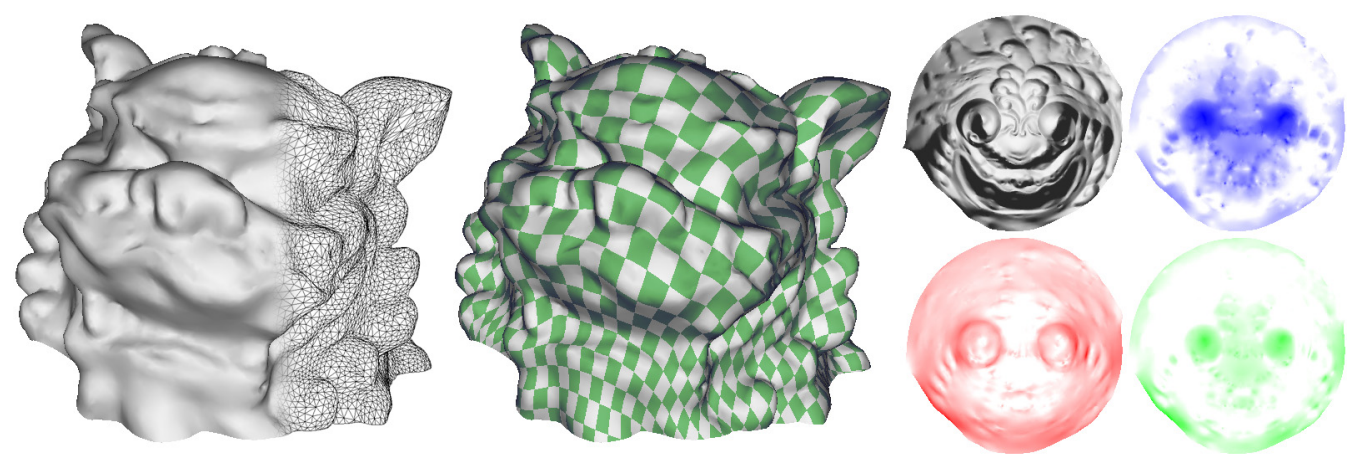

Fig. 1. For a surface mesh (left, 36k triangles), a parameterization is computed, depicted through a texture map (middle). The parameter domain (right) is optimized through the minimization of energy functions which control length, area and angle distortion (shown clockwise from top right; dark regions indicate higher energy).

edge, it was Moser who was able to construct area preserving maps on general manifolds $[13,14]$. In this setting one also faces the problem of distortion, which typically appears as angle distortion.

In this paper we introduce a rigorous variational framework for the construction of low-distortion parameterizations. It is based on the rich mathematical theory built up over decades in the study of rational mechanics [15-17]. Starting with some basic axioms, we derive a unified energy density which allows combined control over angle, area and length distortion in a single, concise framework. We will show in the continuous setting that minimizers of such a parameterization energy exist under suitable assumptions. Thus, we will study general parameterizations as well as those which are constrained in the class of conformal maps and in the class of area preserving maps. We strongly believe that one must study the continuous setting before considering the discrete case: although questions of existence may be simpler to answer when dealing with triangular grids, this approach hides other important questions such as the asymptotic behavior for successively refined grids or the proper choice of constraints. For example, enforcing point constraints is possible on triangular grids, but singularities will be observed under refinement, a phenomenon which is well understood in the continuous setting. Accordingly, we will propose to fix integral quantities in what follows.

There are multiple relationships between our method and earlier parameterization algorithms which share some of the properties with the method presented here. In the overview given by Floater and Hormann [1], they discuss a variational method on continuous surfaces and compare various energy densities. Other previous work in surface parameterization can be distinguished through the different energies that they minimize.

Sander et al. [18] minimize norms of the eigenvalues of the Jacobian similar to our length control energy in Eq. (6). Hormann and Greiner [19] define the MIPS 
energy to minimize the condition number of the Jacobian, using the same local quantity as we do to penalize non-conformality. Degener et al. extend MIPS with a term that penalizes area distortion [20]. Sorkine et al. measure distortion as the maximum eigenvalue of the Jacobian and its inverse [21]. Desbrun et al. [3] derive from a set of axioms similar to ours (cf. Section 3), but lack the axiom of isotropy, a higher order energy in the discrete setting of triangulations. Their derivation involves a conformal energy and a quadratic approximation to the mismatch of the Euler characteristic, i.e., the local sum of angles at the vertices. These quadratic energy terms are closely related to our own conformal energy in Eq. (6).

Lévy et al. [4] minimize discrete conformal energies. Compared to our conformality control in Eq. (6), their energy density is not scale-invariant. Thus length and angle control cannot be steered independently. Sheffer and de Sturler [22] formulate angle deviation directly as a non-linear optimization problem. Here, an energy is considered which is based solely on angle distortion. They use a Newton method to minimize a weighted sum of all these distortions under a large set of constraints to keep the geometry in shape.

An approach to introduce conformal parameterizations on higher genus surfaces is given by $\mathrm{Gu}$ and Yau [5]. In the case of surfaces which are topologically spheres, they stress the fact that for spherical parameterizations harmonicity induces conformality. A generalization of our approach to spherical parameter domains would make our length control energy reflect their Dirichlet energy.

In these approaches the interaction between area, angle and length distortion is not always easily controlled or mathematically well understood, e.g., existence of solutions. We desire a unified framework which incorporates different distortion measurements and their trade-offs while providing clear mathematical statements as to the properties of this energy. In particular the design of stable and scalable numerical solvers, such as those of multigrid type [23], requires an understanding of the mathematical properties of the energy to be minimized.

In what follows, we develop an approach which is mathematically rigorous, providing the different distortion measure controls we desire, and having robust numerical solution methods associated with it. We follow the general procedure in rational mechanics, in particular the mathematical modeling in non-linear elasticity [15-17], where we consider certain natural invariance principles, such as frame indifference and a properly-defined notion of isotropy. In particular, in Section 3 we derive a class of energy functionals from the general principles of elasticity and the axioms of frame indifference and isotropy. We show these to be geometrically meaningful as they allow us to measure area, angle and length distortion in a flexible manner. In Section 4 we will prove existence results for the corresponding parameterizations and after a general discussion of our approach in Section 5, we will discuss the Euler-Lagrange equation which characterizes the minimizer. In Section 7 a straightforward discretization for arbitrary triangular meshes is intro- 
duced and we demonstrate that the resulting discrete minimization problems can be efficiently solved using a constrained Newton method with a suitable step size control. Finally, in Section 9 we draw conclusions.

\section{Notation}

Let $L^{p}(\omega, B)$ with $p \in[1, \infty]$ be the Lebesgue spaces of functions from a domain $\omega$ into some vector space $B$. In the case that $B=\mathbb{R}$ we omit the second parameter. Furthermore, we denote by $H^{1, p}(\omega)$ the usual Sobolev space of functions in $L^{p}(\omega)$ with weak first derivatives also in $L^{p}(\omega)$.

In what follows, we consider a smooth embedded manifold $\mathcal{M} \subset \mathbb{R}^{3}$. For a smooth function $u$ on $\mathcal{M}$ we can define a gradient $\nabla_{\mathcal{M}} u$, which coincides with the projection of the Euclidean gradient in $\mathbb{R}^{3}$ on the tangent space $\mathcal{T}_{x} \mathcal{M}$ for functions $u$ which extend to a neighborhood of $\mathcal{M}$ in $\mathbb{R}^{3}$. For the vector-valued mapping $u: \mathcal{M} \rightarrow \mathbb{R}^{2}$ we define the gradient of $u$ as the tensor whose rows are the gradients of the components of $u$.

Suppose $(x, \omega)$ is an initial parameterization of an open, bounded and connected subset $\Omega \subset \mathcal{M}$. Furthermore, we assume that $x: \bar{\omega} \rightarrow \mathcal{M} ; \xi \mapsto x(\xi)$ is a diffeomorphism from the closure $\bar{\omega}$ of $\omega$ onto the closure $x(\bar{\omega})=\bar{\Omega}$ of the surface patch $\Omega$. Later the regularity assumptions will be weakened (cf. Theorem 4.2). In

$x=x(\xi)$ on $\mathcal{M}$ the tangent space $\mathcal{T}_{x} \mathcal{M}$ is spanned by the basis $\left\{\frac{\partial x}{\partial \xi_{1}}, \frac{\partial x}{\partial \xi_{2}}\right\}$. Measuring length on $\mathcal{M}$ requires the definition of a metric $g$. In matrix notation we obtain $g=\left(g_{k l}\right)$ with

$$
g_{k l}=\frac{\partial x}{\partial \xi_{k}} \cdot \frac{\partial x}{\partial \xi_{l}},
$$

where "." indicates the scalar product in $\mathbb{R}^{3}$. It is well known that the metric $g$ defines a scalar product " $\cdot_{g}$ " with $v \cdot_{g} w:=g v \cdot w$ on $\omega$, where here "." is the standard scalar product on $\mathbb{R}^{2}$. This new product is the pull-back of the scalar product of tangent vectors $D x v$ and $D x w$ on $\mathcal{T}_{x} \mathcal{M}$ for $v, w \in \mathbb{R}^{2}$, where $D x \in$ $\mathbb{R}^{3,2}$ is the derivative of the parameterization $x$. As in the standard case of the Euclidean scalar product, one can define for any matrix $A \in \mathbb{R}^{2,2}$ a $g$-adjoint map $A^{*} \in \mathbb{R}^{2,2}$ by

$$
g A v \cdot w=g v \cdot A^{*} w
$$

for all $v, w \in \mathbb{R}^{2}$. It follows that $A^{*}=g^{-1} A^{T} g$. Indeed, we obtain $g A v \cdot w=$ $A v \cdot g w=g^{-1} g v \cdot A^{T} g w=g v \cdot g^{-1} A^{T} g w$. A matrix $A \in \mathbb{R}^{2,2}$ is called $g$-self adjoint if $A^{*}=A$ and $g$-orthogonal if $A^{*}=A^{-1}$. 

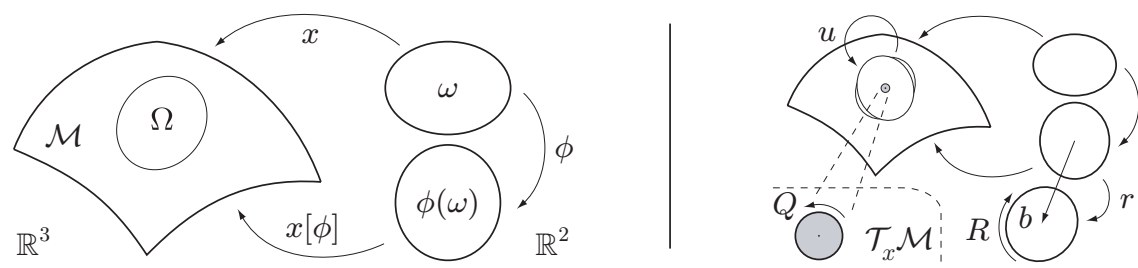

Fig. 2. The setup of our parameterization with a given initial chart $x$ and the deformed chart $x[\phi]$; a rigid body motion $r: \xi \mapsto R \xi+b$ of the deformed parameter domain $\phi(\omega)$ and a smooth mapping $u$ from $\mathcal{M}$ onto itself, which at $x \in \mathcal{M}$ is locally a rotation $Q$ on $\mathcal{T}_{x} \mathcal{M}$.

\section{A Variational Formulation Derived From First Principles}

To measure the local distortion of a parameterization $x$, we define the distortion tensor as the intrinsic gradient $\nabla_{\mathcal{M}} y$ of the mapping $y: \mathcal{M} \rightarrow \mathbb{R}^{2}$, where $y=x^{-1}$ denotes the inverse of the parameter mapping $x$ from the surface into the parameter domain at some point $x$ on $\mathcal{M}$. As a linear mapping, $\nabla_{\mathcal{M}} y$ maps tangent vectors $w \in \mathcal{T}_{x} \mathcal{M}$ to vectors in $\mathbb{R}^{2}$,

$$
\left(\nabla_{\mathcal{M}} y\right) w=\frac{d}{d t}(y \circ c)(0)
$$

where $c$ is a curve on $\mathcal{M}, c(0)=x$ and $\dot{c}(0)=w$. To motivate our definition of distortion, we replace for a moment the surface patch $\Omega$ on $\mathcal{M}$ by a Euclidean domain $\Omega \subset \mathbb{R}^{2}$. A fundamental assumption from the theory of elasticity $[15,16]$ tells us that all distortion measures for a given deformation $\phi: \Omega \rightarrow \mathbb{R}^{2}$, such as length, area, or angle distortion, can be expressed in terms of the deformation tensor $\nabla \phi$. Thus measuring the distortion of a mapping is directly linked to measuring the elastic energy of the associated deformation. In our setting we replace the elastic body $\Omega \subset \mathbb{R}^{2}$ by our surface patch $\Omega \subset \mathcal{M}$ and consider parameterizations to be elastic surface deformations. What remains is to select a suitable energy which depends on $\nabla_{\mathcal{M}} y$. The choice of this energy will be guided by fixing suitable axioms (see below) and the desired parameterization will follow as the minimizer of this energy.

Instead of optimizing the mapping $x^{-1}$ directly, we will consider a Euclidean deformation $\phi: \omega \rightarrow \mathbb{R}^{2}$ of an initial parameter domain $\omega$ which is a diffeomorphism. On the deformed domain $\tilde{\omega}:=\phi(\omega)$ a new parameterization

$$
\tilde{x}=x[\phi]:=x \circ \phi^{-1}
$$

is given. Hence, one can rephrase the goal of finding an optimal parameterization in terms of deformations of a given parameterization and ask for an optimal deformation of the latter (cf. Fig. 2). Obviously, any smooth parameterization $\tilde{x}$ of the surface patch $\Omega$ can be written as $\tilde{x}=x[\phi]$ for $\phi:=\tilde{x}^{-1} \circ x$. Optimality will be 
expressed in terms of deformations minimizing a suitable energy $E$ on a space of admissible deformations $\mathcal{A}$. We will now state some assumptions on this energy:

- Elasticity. The energy is elastic, in particular it is translation invariant and locally on $\mathcal{M}$ depends solely on the distortion tensor $\nabla_{\mathcal{M}} x^{-1}(\cdot)$ of the deformation from $\Omega \subset \mathcal{M}$ onto the deformed parameter domain $\phi(\omega)$. Here, $x^{-1}[\phi]=\tilde{x}^{-1}=$ $\phi \circ x^{-1}$. In particular, the energy density does not depend on the history of the deformation, nor on higher deformation derivatives. Physically, we might think of our surface as a thin shell which we press flat between two plates. One can distinguish between stresses induced by stretching, shearing and other deformations, and stresses induced by bending that occurs in the surface as it is being pressed. Our deformation $x[\phi]$ can be regarded as the flattening of such a thin shell. Then the elastic energy considered here reflects the stresses in the plane but not the bending of the normals orthogonal to the plane. The latter would lead to an energy which depends on higher derivatives of the deformation.

We suppose that the local energy depends solely on the first order variation of the deformation. In fact, we assume that there exists an energy density $W_{\mathcal{M}}$ : $\mathcal{T} \mathcal{M}^{2} \rightarrow \mathbb{R}$ such that

$$
E[\phi]=\int_{\Omega} W_{\mathcal{M}}\left(\nabla_{\mathcal{M}} x^{-1}[\phi]\right) \mathrm{d} A .
$$

We can rewrite this in terms of the Euclidean deformation $\phi$ as

$$
E[\phi]=\int_{\omega} \hat{W}(D \phi) \sqrt{\operatorname{det} g} \mathrm{~d} \xi
$$

where $\hat{W}(A):=W_{\mathcal{M}}\left(A \nabla_{M} x^{-1}\right)$ for any $A \in G L(2)$.

- Frame indifference. The energy does not depend on rigid body motions $\xi \mapsto$ $R \xi+b$ of the parameter domain $\phi(\omega)$ with $R \in S O(2)$ and $b \in \mathbb{R}^{2}$ (cf. Fig. 2), i.e. at $x$ on $\mathcal{M}$ we require

$$
W_{\mathcal{M}}\left(\nabla_{\mathcal{M}} r \circ x^{-1}[\phi]\right)=W_{\mathcal{M}}\left(\nabla_{\mathcal{M}} x^{-1}[\phi]\right)
$$

and hence for $\hat{W}$ and all $A \in G L(2)$ we obtain

$$
\hat{W}(R A)=\hat{W}(A) .
$$

- Isotropy. The energy does not depend on directions on $\mathcal{M}$. If $u: \mathcal{M} \rightarrow \mathcal{M}$ is any smooth mapping from $\mathcal{M}$ onto itself which at any point $x \in \mathcal{M}$ is locally a rotation, then the energy density at the point $x$ is not affected by this transformation (cf. Fig. 2), i.e. at $x$ on $\mathcal{M}$ we require

$$
W_{\mathcal{M}}\left(\nabla_{\mathcal{M}}(u \circ x[\phi])^{-1}\right)=W_{\mathcal{M}}\left(\nabla_{\mathcal{M}} x^{-1}[\phi]\right) .
$$

In terms of $\hat{W}$ we obtain that for all $g$-orthogonal matrices $Q$ and all $A \in G L(2)$

$$
\hat{W}(A Q)=\hat{W}(A) .
$$


Based on these "first principles" we will now derive a simple form of the energy. Furthermore, the free parameters of the resulting energy density will have a clear geometric interpretation. For a matrix $A \in \mathbb{R}^{2,2}$ we denote the principal invariants by $\iota_{A}:=(\operatorname{tr}(A), \operatorname{det}(A))$, where $\iota_{(\cdot)}: \mathbb{R}^{2,2} \rightarrow R^{2}$. The following theorem is an adaptation of the famous Rivlin-Ericksen representation theorem in non-linear elasticity to our geometric setting (cf. also Theorem 4.4-1 [16]). As in this classical result from rational mechanics, the remaining free parameters are those we intuitively already expect. In particular, the above theorem precisely refers to the assumptions that we have made.

Theorem 3.1 (Representation of the energy) Suppose $\mathcal{M}$ is a smooth surface, and $x$ is an initial parameterization of an open set $\Omega \subset \mathcal{M}$ over a bounded, open set $\omega \subset \mathbb{R}^{2}$. We assume that $x$ is actually defined on $\bar{\omega}$ and it is a diffeomorphism onto $\bar{\Omega}$. Let the assumptions of elasticity (1), frame indifference (2) and isotropy (3) hold. Then there exists a function $W: \mathbb{R}^{2} \rightarrow \mathbb{R}$ such that $\hat{W}(A)=W\left(\iota_{g^{-1}} A^{T} A\right)$ and thus

$$
E[\phi]=\int_{\omega} W\left(\operatorname{tr}\left(D \phi g^{-1} D \phi^{T}\right),(\operatorname{det} D \phi)^{2}(\operatorname{det} g)^{-1}\right) \sqrt{\operatorname{det} g} d \xi
$$

Proof Due to our assumptions, $g^{-1}$ is symmetric, uniformly positive definite and uniformly bounded. Suppose $A$ is an arbitrary invertible matrix in $\mathbb{R}^{2,2}$. By the polar decomposition theorem there exists an orthogonal matrix $Q$ such that $A=Q U$ where $U=\left(A^{T} A\right)^{\frac{1}{2}}$ is symmetric and positive definite. Hence, by the assumption of frame indifference (2) we obtain

$$
\hat{W}(A)=\hat{W}(Q U)=\hat{W}(U)=\hat{W}\left(\left(A^{T} A\right)^{1 / 2}\right) .
$$

Next, we define $\tilde{W}(C):=\hat{W}\left(C^{\frac{1}{2}}\right)$ for all symmetric, positive definite matrices $C$ and obtain

$$
\hat{W}(A)=\tilde{W}\left(A^{T} A\right)
$$

From this and the assumption of isotropy (3) one deduces

$$
\tilde{W}\left(A^{T} A\right)=\hat{W}(A)=\hat{W}(A Q)=\tilde{W}\left(Q^{T} A^{T} A Q\right)
$$

for all $g$-orthogonal matrices $Q$. In particular, for symmetric, positive definite matrices $C$ we get

$$
\tilde{W}(C)=\tilde{W}\left(Q^{T} C Q\right)
$$


Next, we show that whenever $\iota_{g^{-1} A}=\iota_{g^{-1} B}$ for symmetric, positive definite matrices $A, B \in \mathbb{R}^{2,2}$, then $\tilde{W}(A)=\tilde{W}(B)$. Thus, we immediately deduce that there is a function $W$ such that $\tilde{W}(A)=W\left(\iota_{g^{-1} A}\right)$ and hence for general matrices $A \in \mathbb{R}^{2,2}$,

$$
\hat{W}(A)=\tilde{W}\left(A^{T} A\right)=W\left(\iota_{g^{-1} A^{T} A}\right)
$$

which is the assertion in our theorem.

First, we observe that for any symmetric matrix $A$, the matrix $g^{-1} A$ is $g$-self adjoint. Indeed, $\left(g^{-1} A\right)^{*}=g^{-1} A^{T} g^{-1} g=g^{-1} A$. Thus, we deduce that $g^{-1} A$ and $g^{-1} B$ diagonalize with respect to the scalar product ". $g$ " and we obtain

$$
\begin{aligned}
& g^{-1} A=\lambda_{1} r_{1}\left(g r_{1}\right)^{T}+\lambda_{2} r_{2}\left(g r_{2}\right)^{T} \\
& g^{-1} B=\mu_{1} s_{1}\left(g s_{1}\right)^{T}+\mu_{2} s_{2}\left(g s_{2}\right)^{T}
\end{aligned}
$$

with $g r_{i} \cdot r_{j}=\delta_{i j}$ and $g s_{i} \cdot s_{j}=\delta_{i j}$. Due to our assumption $\iota_{g^{-1} A}=\iota_{g^{-1} B}$ we achieve $\mu_{i}=\lambda_{i}$ after a possible reordering. Furthermore, there exists a $g$-orthogonal matrix $Q$ with $Q s_{i}=r_{i}$. Thus we get

$$
\begin{aligned}
A & =g g^{-1} A=\sum_{i=1,2} \lambda_{i} g Q s_{i}\left(g Q s_{i}\right)^{T}= \\
& =\sum_{i=1,2} \lambda_{i} g Q s_{i} s_{i}^{T} Q^{T} g=\sum_{i=1,2} \lambda_{i} Q^{-T} g s_{i} s_{i}^{T} g Q^{-1} \\
& =Q^{-T} g \sum_{i=1,2} \lambda_{i} s_{i}\left(g s_{i}\right)^{T} Q^{-1}=Q^{-T} g g^{-1} B Q^{-1} \\
& =Q^{-T} B Q^{-1} .
\end{aligned}
$$

Here we have used the fact that $g Q^{-1}=Q^{T} g$ holds for $g$-orthogonal matrices $Q$ and that $Q^{-1}$ is $g$-orthogonal if $Q$ is $g$-orthogonal, which implies $g Q=g\left(Q^{-1}\right)^{-1}=$ $Q^{-T} g$. Now, Eq. (5) applied tothe $g$-orthogonal matrix $Q^{-1}$ leads to

$$
\tilde{W}(A)=\tilde{W}\left(Q^{-T} B Q^{-1}\right)=\tilde{W}(B),
$$

which is what we have claimed above. Finally, we obtain for the principal invariants

$$
\begin{aligned}
\operatorname{tr}\left(g^{-1} D \phi^{T} D \phi\right) & =\operatorname{tr}\left(D \phi g^{-1} D \phi^{T}\right), \\
\operatorname{det}\left(g^{-1} D \phi^{T} D \phi\right) & =(\operatorname{det} g)^{-1}(\operatorname{det} D \phi)^{2} .
\end{aligned}
$$

Let us give a geometric interpretation of this result. For a given smooth and injective deformation $\phi: \omega \rightarrow \mathbb{R}^{2}$ with inverse $\psi=\phi^{-1}$ we have already considered the new parameterization $(\tilde{x}, \tilde{\omega})$ with 


$$
\tilde{x}(\tilde{\xi})=x[\phi](\tilde{\xi})=(x \circ \psi)(\tilde{\xi}) .
$$

From $\frac{d}{d \tilde{\xi}}=\frac{d \xi}{d \tilde{\xi}} \frac{d}{d \xi}=D \psi \frac{d}{d \xi}$ we obtain the push-forward of the metric $g_{i j}$ on $\tilde{\omega}$,

$$
g[\phi]:=\tilde{g}=D \psi^{T} g D \psi .
$$

The inverse $g^{-1}[\phi]:=\tilde{g}^{-1}=\left(\tilde{g}^{i j}\right)_{i j}$ is then given by

$$
\begin{aligned}
g^{-1}[\phi] & =\left((D x D \psi)^{T} D x D \psi\right)^{-1}=\left(D \psi^{T} D x^{T} D x D \psi\right)^{-1} \\
& =D \phi g^{-1} D \phi^{T}
\end{aligned}
$$

Thus, $\iota_{g^{-1}[\phi]}=\iota_{D \phi g^{-1} D \phi^{T}}=\iota_{g^{-1} D \phi^{T} D \phi}$ and our representation theorem above implies that the energy integrand depends on the principal invariants of the inverse metric $g^{-1}[\phi]$ of the deformed configuration. Obviously, $g^{-1}[\phi]$ is symmetric positive definite. We denote its eigenvalues by $\Gamma$ and $\gamma$, where we assume $\Gamma \geq \gamma$.

If we consider the Frobenius norm $\|A\|=\sqrt{\operatorname{tr} A^{T} A}$ for linear mappings then the pull-back norm on the space of linear maps from $\mathcal{T}_{x} \mathcal{M} \rightarrow \mathcal{T}_{\tilde{x}} \mathcal{M}$ is given by $\|A\|_{g^{-1}}=\sqrt{A g^{-1} A^{T}}$. Hence, just as $\sqrt{\operatorname{tr}\left(A^{T} A\right)}$ measures the average change of length under a linear mapping $A,\|D \phi\|_{g^{-1}}=\sqrt{\operatorname{tr}\left(D \phi g^{-1} D \phi^{T}\right)}$ measures the average length change of the tangent vectors under the inverse parameterization $x^{-1}[\phi]$. Note that Theorem 3.1 implies that we are not able to distinguish length change in different directions separately, mainly due to the postulated isotropy assumption. Additionally, $\sqrt{\operatorname{det}\left(g^{-1}[\phi]\right)}$ measures the change of area under the inverse map $x^{-1}[\phi]$. This map is locally area preserving if $\operatorname{det}\left(g^{-1}[\phi]\right)=1$. In terms of the eigenvalues $\Gamma, \gamma$ we obtain:

$$
\begin{aligned}
& a=\operatorname{tr}\left(g^{-1}[\phi]\right)=\Gamma+\gamma, \\
& d=\operatorname{det}\left(g^{-1}[\phi]\right)=\Gamma \gamma .
\end{aligned}
$$

In fact, the eigenvalues can be expressed in terms of the invariants:

$$
\Gamma=\frac{a}{2}+\sqrt{\frac{a^{2}}{4}-d}, \quad \gamma=\frac{a}{2}-\sqrt{\frac{a^{2}}{4}-d} .
$$

The condition for conformality is $\Gamma=\gamma$. Thus, $(\Gamma-\gamma)^{2}=a^{2}-4 d=0$ is an indication for local conformality. This expression is not invariant w.r.t. simple scaling. Therefore we propose to replace it by the expression

$$
(\Gamma-\gamma)^{2} / d=\frac{\Gamma}{\gamma}+\frac{\gamma}{\Gamma}-2=a^{2} / d-4
$$


which is scale invariant. It follows that these are natural quantities for an energy measuring the quality of parameterizations. The representation theorem states that under our assumptions an energy density depending on these quantities already reflects the most general case. Now, we are going to discuss the actual quality of a parameterization $x[\phi]$ in terms of the energy integrand $W$. We are aiming for minimal distortion of length, area and angles. One easily verifies (cf. $[15,16])$ that length is strictly preserved if $x[\phi]$ is an isometry $(g[\phi]=\mathbb{I})$ and thus

$$
D \phi g^{-1} D \phi^{T}=\mathbb{I}
$$

Precise area preservation is ensured if $(\operatorname{det} D \phi)^{2}(\operatorname{det} g)^{-1}=1$. Thus, we propose a concrete energy density

$$
W(a, d)=\alpha_{l} a^{\frac{p}{2}}+\alpha_{a}\left(d^{\frac{r}{2}}+\beta d^{-\frac{s}{2}}\right)+\alpha_{c}\left(a^{2} / d-4\right)^{t}
$$

with $a=a(A)=\operatorname{tr}\left(A g^{-1} A^{T}\right)$ and $d=d(A)=\operatorname{det}(A)^{2} \operatorname{det} g^{-1}$, which takes care of all our distortion quantities. This energy can be regarded as a prototype of an energy density which is in accordance with Theorem 3.1 and reflects the convexity and growth conditions required for the existence result in Theorem 4.2 below. Here, $\alpha_{l} a^{\frac{p}{2}}$ controls the length change of the inverse parameterization $x^{-1}[\phi]$, whereas $\alpha_{a}\left(d^{\frac{r}{2}}+\beta d^{-\frac{s}{2}}\right)$ penalizes area compression and expansion of the parameterization $x[\phi]$, respectively. Finally, $\alpha_{c}\left(a^{2} / d-4\right)^{t}$ controls the lack of conformality. In particular, we have seen above that $a^{2} / d-4=0$ holds for conformal parameterizations. This energy is not convex, but it is polyconvex [24] (i.e., a function $\hat{F}(A)$ is called polyconvex if $\hat{F}(A)=F(A$, $\operatorname{det} A)$ with $F$ convex in both arguments). It is a specific, suitable model from a larger class of functionals for which we will prove the existence of a minimizing deformation in a suitable class of admissible deformations.

It is reasonable to ask that an isometry is an optimal parameterization. From this requirement we deduce the following restriction on the parameters:

Lemma 3.2 Suppose $\mathcal{M}$ is a smooth surface, and $x$ is an initial parameterization of an open set $\Omega \subset \mathcal{M}$ over a bounded, open set $\omega \subset \mathbb{R}^{2}$. We assume that $x$ is defined on $\bar{\omega}$ and it is a diffeomorphism onto $\bar{\Omega}$. A deformation $\phi$ which turns the parameterization $x[\phi]$ into an isometry is a local minimizer of the elastic energy $E[\cdot]$ with the density defined in Eq. (6) if

$$
\begin{aligned}
& p \geq 2, \quad r, s \geq 0, \quad t \geq 2 \text { or } t=1, \quad \alpha_{l}, \alpha_{a}>0, \quad \alpha_{c} \geq 0 \\
& \alpha_{l} 2^{\frac{p}{2}-1} p+\alpha_{a}(r-\beta s)=0 .
\end{aligned}
$$

Proof Let us take derivatives of the energy integrand $W$ with respect to the inverse metric $q=A g^{-1} A^{T}$ in the direction of an arbitrary symmetric matrix $C \in \mathbb{R}^{2,2}$ : 


$$
\begin{aligned}
& \partial_{q} W\left(\iota_{q}\right)(C)=\alpha_{l} \frac{p}{2} \operatorname{tr}(q)^{\frac{p}{2}-1} \operatorname{tr}(C)+\alpha_{a}\left(\frac{r}{2}(\operatorname{det} q)^{\frac{r}{2}}-\beta \frac{s}{2}(\operatorname{det} q)^{-\frac{s}{2}}\right) \operatorname{tr}\left(q^{-1} C\right) \\
& \quad+\alpha_{c} t\left(\frac{\operatorname{tr}(q)^{2}}{\operatorname{det}(q)}-4\right)^{t-1}\left(2 \frac{\operatorname{tr}(q)}{\operatorname{det}(q)} \operatorname{tr}(C)-\frac{\operatorname{tr}(q)^{2}}{\operatorname{det}(q)} \operatorname{tr}\left(q^{-1} C\right)\right), \\
& \partial_{q}^{2} W\left(\iota_{q}\right)(C, C)=\alpha_{l} \frac{p}{2}\left(\frac{p}{2}-1\right) \operatorname{tr}(q)^{\frac{p}{2}-2} \operatorname{tr}(C)^{2} \\
& +\alpha_{a}\left(\left(\frac{r}{2}\right)^{2}(\operatorname{det} q)^{\frac{r}{2}}+\beta\left(\frac{s}{2}\right)^{2}(\operatorname{det} q)^{-\frac{s}{2}}\right) \operatorname{tr}\left(q^{-1} C\right)^{2} \\
& +\alpha_{a}\left(\beta \frac{s}{2}(\operatorname{det} q)^{-\frac{s}{2}}-\frac{r}{2}(\operatorname{det} q)^{\frac{r}{2}}\right) \operatorname{tr}\left(q^{-1} C q^{-1} C\right) \\
& +\alpha_{c} t\left(\frac{\operatorname{tr}(q)^{2}}{\operatorname{det}(q)}-4\right)^{t-1}\left(2 \frac{\operatorname{tr}(C)^{2}}{\operatorname{det}(q)}-2 \frac{\operatorname{tr}(q) \operatorname{tr}(C) \operatorname{tr}\left(q^{-1} C\right)}{\operatorname{det}(q)}\right. \\
& \left.\quad+\frac{\operatorname{tr}(q)^{2}}{\operatorname{det}(q)} \operatorname{tr}\left(q^{-1} C\right)^{2}+\frac{\operatorname{tr}(q)^{2}}{\operatorname{det}(q)} \operatorname{tr}\left(q^{-1} C q^{-1} C\right)\right) \\
& +\alpha_{c} t(t-1)\left(\frac{\operatorname{tr}(q)^{2}}{\operatorname{det}(q)}-4\right)^{t-2}\left(2 \frac{\operatorname{tr}(q)}{\operatorname{det}(q)} \operatorname{tr}(C)-\frac{\operatorname{tr}(q)^{2}}{\operatorname{det}(q)} \operatorname{tr}\left(q^{-1} C\right)\right)^{2}
\end{aligned}
$$

Under our usual assumptions every diffeomorphism $\phi: \omega \rightarrow \phi(\omega)$ induces a positive definite inverse metric $q=g^{-1}[\phi]$. Hence, $q=\mathbb{I}$ is a local minimizer of $W\left(\iota_{q}\right)$ if $\partial_{q} W\left(\iota_{\mathbb{I I}}\right)=0$ and $\partial_{q}^{2} W\left(\iota_{\mathbb{I I}}\right)$ is positive definite. This leads to

$$
\begin{aligned}
\partial_{q} W\left(\iota_{\mathbb{I I}}\right)(C)= & \frac{1}{2}\left(\alpha_{l} 2^{\frac{p}{2}-1} p+\alpha_{a}(r-\beta s)\right) \operatorname{tr}(C)=0, \\
\partial_{q}^{2} W\left(\iota_{\mathrm{II}}\right)(C, C)= & \frac{1}{2}\left(\alpha_{l} p\left(\frac{p}{2}-1\right) 2^{\frac{p}{2}-2}+\alpha_{a}\left(\frac{r^{2}}{4}+\beta \frac{s^{2}}{4}\right)\right) \operatorname{tr}(C)^{2} \\
& +\frac{1}{2} \alpha_{a}(\beta s-r) \operatorname{tr}\left(C^{2}\right)+6 \alpha_{c} t \delta(t-1) \operatorname{tr}\left(C^{2}\right)>0,
\end{aligned}
$$

for all symmetric matrices $C \neq 0$, with $\delta(s)=1$ if $s=0$, otherwise $\delta(s)=0$. Here we have used the fact that $\operatorname{tr}\left(C^{2}\right)>0$ for any symmetric matrix $C$ with $C \neq 0$, and we have taken into account the preceding equation to obtain the last inequality above.

\section{Existence of minimizing deformations}

So far, we have not taken into account the invariance of the energy with respect to rigid body motions due to our assumption on frame indifference (2). Indeed, if we do not enforce additional restrictions on the minimizing deformation $\phi$, we can only expect an optimal parameterization to be given up to rigid body motions in $\mathbb{R}^{2}$ and any $\hat{\phi}(\xi)=R \phi(\xi)+b$ with $R \in S O(2)$ and $b \in \mathbb{R}^{2}$ also minimizes $E$. To overcome this ambiguity we require admissible deformations to have a fixed zero moment and in addition we fix the angular momentum, i.e., we consider $\phi$ with 


$$
\begin{aligned}
& M_{0}(\phi):=\int_{\omega} \phi(\xi) \mathrm{d} \xi=0 \\
& M_{1}(\phi):=\int_{\omega} \phi(\xi)_{2} \xi_{1}-\phi(\xi)_{1} \xi_{2} \mathrm{~d} \xi=0 .
\end{aligned}
$$

Lemma 4.1 Let $\phi$ and $\hat{\phi}$ be as above, i.e., they only differ by a rigid body motion. If $M_{0}(\phi)=M_{0}(\hat{\phi})=0$ and $M_{1}(\phi)=M_{1}(\hat{\phi})=0$ then either $\phi=\hat{\phi}$ or $\phi=R^{\pi} \hat{\phi}$, where $R^{\pi}$ is a rotation by $\pi$.

Proof If the zero moment $M_{0}$ vanishes, the rigid body motion is a rotation. Therefore it may be represented as $\hat{\phi}(x)=\exp (\alpha Z) \phi(x)$, where

$$
Z=\left(\begin{array}{cc}
0 & -1 \\
1 & 0
\end{array}\right)
$$

and $\alpha \in \mathbb{R}$. The condition $M_{1}(\hat{\phi})=0$ implies

$$
\begin{aligned}
0 & =\int_{\omega} \exp (\alpha Z) \phi(\xi) \cdot Z \xi \mathrm{d} \xi=-\int_{\omega} Z \exp (\alpha Z) \phi(\xi) \cdot \xi \mathrm{d} \xi \\
& =\frac{d}{d \alpha} \int_{\omega} \exp (\alpha Z) \phi(\xi) \cdot \xi \mathrm{d} \xi .
\end{aligned}
$$

From this we deduce $\alpha=0$ or $\alpha=\pi$.

Let us emphasize that due to the non-existence of an embedding $H^{1,2}$ into $C^{0}$ in two dimensions we have to avoid point constraints, and therefore we choose to fix the integral quantities above. Even in the case of higher integrability of the gradients it is preferable to consider integral quantities, since they improve the stability and robustness of the numerical algorithm.

Based on these preliminaries we can now formulate the following existence result:

Theorem 4.2 (Existence of an optimal parameterization) Suppose $\mathcal{M}$ is a Lipschitz continuous surface and $\Omega$ is a bounded, open, connected subset of $\mathcal{M}$, such that $\bar{\Omega}$ is the image under a bi-Lipschitz continuous initial parameterization $x$ over the closure of a set $\omega$ with Lipschitz boundary. Consider the energy

$$
E[\phi]=\int_{\omega} W\left(\operatorname{tr}\left(D \phi g^{-1} D \phi^{T}\right),(\operatorname{det} D \phi)^{2}(\operatorname{det} g)^{-1}\right) \sqrt{\operatorname{det} g} d \xi
$$

(cf. Eq. (4)) acting on deformations from the set of admissible deformations 


$$
\begin{aligned}
\mathcal{A}:=\left\{\phi: \omega \rightarrow \mathbb{R}^{2} \mid \phi\right. & \in H^{1, p}\left(\omega, \mathbb{R}^{2}\right), \operatorname{det} D \phi \in L^{r}(\omega), \\
& \operatorname{det} D \phi>0 \text { a.e. in } \omega, \phi \text { obeys Eq. (7) and Eq. (8) }\}
\end{aligned}
$$

with $p \geq 2, r>1$, and $s>0$. Furthermore, we assume $W(\cdot, \cdot)$ to be convex in both arguments and that the following growth conditions hold:

$$
W(a, d) \geq \alpha_{l} a^{\frac{p}{2}}+\alpha_{a}\left(d^{\frac{r}{2}}+\beta d^{-\frac{s}{2}}\right),
$$

where $\alpha_{l}, \alpha_{a}, \beta>0$. Then there exists a minimizing deformation $\phi$ in $\mathcal{A}$. If in addition we assume that $p>2, s>\frac{p}{p-2}$ and require the constraint

$$
\int_{\Omega} \sqrt{\operatorname{det} g^{-1}[\phi]} d A \leq \operatorname{area}\left(x^{-1}[\phi](\Omega)\right)
$$

to be fulfilled for admissible deformations $\phi$, then the minimizer $\phi$ is continuous and there exists a continuous deformation $\psi \in H^{1, \sigma}(\phi(\omega))$ with $\sigma=\frac{p(1+s)}{s+p}$, which is almost everywhere the inverse of $\phi$.

Before we give a proof of the theorem, let us comment on the analytic background. The growth condition with respect to $\operatorname{det} g^{-1}[\phi]$ ensures the local injectivity of the deformation. Thus local overfolding in the interior of the parameter domain is already ruled out. The additional constraint stated in the theorem prevents the domain image from undergoing a (global) overfolding. Indeed, for general deformations we get from the area formula (cf. Ambrosio et al. [25, Theorem 2.71]) that

$$
\int_{\Omega} \sqrt{\operatorname{det} g^{-1}[\phi]} \mathrm{d} A=\int_{\phi(\omega)} \operatorname{card} \phi^{-1}(\tilde{\xi}) \mathrm{d} \tilde{\xi}
$$

Hence, with this constraint we enforce that $\operatorname{card} \phi^{-1}(\tilde{\xi})=1$ for almost every $\tilde{\xi} \in \phi(\omega)$. Nevertheless self-contact on the boundary of the deformed domain $\phi(\omega)$ is still possible. Unfortunately, this condition is very difficult to control and to implement algorithmically. Practically, if we detect a loss of global injectivity due to overfolding, we should allow the initial parameterization to be split into a finite number of pieces. Then, we restart and expect to obtain smaller optimal parameterizations which are not self-intersecting.

In $[26,27]$ the minimizing deformation is proven to be a homeomorphism under the same growth conditions we use here. The homeomorphism argument is crucial at the boundary, where Ball [26] requires Dirichlet boundary data, which is already given as the trace of a homeomorphism and the domain is assumed to fulfill the cone condition. Due to the application we have in mind, we have to deal with Neumann boundary conditions. Hence, we have no strong knowledge on the regularity of the deformed boundary. This makes it difficult to obtain the full regularity stated in [26]. 
Proof [Theorem 4.2] Based on a nowadays classical result in the calculus of variations the proof is quite straightforward. Let us briefly sketch the main ingredients. We follow the well-known procedure of the direct methods in the calculus of variations $[7,24,28]$, in particular the proof of the existence of minimizers for such polyconvex energy functions by Ball [29] (cf. also the monograph by Ciarlet [16], Theorem 7.7-1). Here, we only give a brief summary of the arguments. From the biLipschitz assumption on the initial parameterization we deduce that $g^{-1} \in L^{\infty}(\omega)$ and it is uniformly positive definite. Hence, $\sqrt{\operatorname{tr}\left(A g^{-1} A^{T}\right)}$ is actually a norm on linear mappings $A \in \mathbb{R}^{2,2}$.

The growth conditions imply that for every constant $C$ there exists a ball $B_{R}(0)$ in $\mathcal{A}$ with radius $R$ such that $E[\phi]>C$ outside $B_{R}(0)$. Thus, there exists a minimizing sequence $\left(\phi^{k}\right)_{k \in \mathbb{N}}$ with $\left\|D \phi^{k}\right\|_{L^{p}(\omega)}$ and $\left\|\operatorname{det} D \phi^{k}\right\|_{L^{r}(\omega)}$ being uniformly bounded. Due to the constraint on the zero moment and a generalized Poincaré inequality, the $H^{1, p}(\omega)$ norm of $\phi^{k}$ also is uniformly bounded. Because of the reflexivity of $H^{1, p}(\omega) \times L^{r}(\omega)$ for $p, r>1$ we can extract a weakly convergent subsequence from the minimizing sequence.

Applying well-known results on the weak continuity of the determinant we get that $\left(\phi^{k}, \operatorname{det} D \phi^{k}\right)$ converges weakly to $(\phi, \operatorname{det} D \phi)$. Furthermore, Ball proved that for the limit deformation det $D \phi>0$ holds almost everywhere in $\omega$ for $s>0$. Due to the convexity of $W$ in both arguments we finally obtain a result on weak sequentially lower semicontinuity and thus establish the existence of a minimizing deformation in $\mathcal{A}$. By Sobolev's embedding theorem we claim that $\phi \in C^{0}(\bar{\Omega})$ if $p>2$. In the constrained case, we follow Ciarlet and Necas [30] and use the weak continuity of det $D \phi$ to prove that the set of admissible deformations including the additional constraint is weakly closed. In particular we obtain that the deformation $\phi$ is almost everywhere invertible. The existence and the stated properties of the function $\psi$ can be derived using the arguments by Ball [26]. First, one verifies that the candidate for the gradient of the inverse $(D \phi)^{-1}$ is in $L^{\sigma}$ with $\sigma=\frac{p(1+s)}{s+p}$. This follows immediately from the growth conditions by Hölder's inequality. Furthermore, one verifies that $\sigma>2$. Then, following Ball we are able to prove that an inverse $\psi$ exists almost everywhere and its gradient at the deformed position is actually $(D \phi)^{-1}$. Hence, $\psi \in H^{1, \sigma}$ and again by Sobolev's embedding theorem $\psi$ is continuous.

Remark 4.3 (Generalizing to higher dimensions) The approach presented here is not restricted to two-dimensional surfaces. Following the guidelines on nonlinear elasticity $[15,16,24,29]$ one can easily generalize these results to higher dimensions $n>2$. Besides length and volume control, a control of the average area change of all types of immersed $n-k$ dimensional surfaces for $k=2, \cdots, n-1$ can be taken into account. This will be reflected in the energy by corresponding norms of all minors of $g^{-1}[\phi]$. Due to the applications we have in mind here, we restrict ourselves to the case of two-dimensional surfaces embedded in $\mathbb{R}^{3}$. 
One might ask for explicitly area preserving parameterizations, and thus request that $\operatorname{det} g^{-1}[\phi]=1$. Indeed, there is the following modified existence result for such deformations.

Corollary 4.4 (Existence of an optimal, area preserving parameterization) Under the assumptions of Theorem 4.2 with $\mathcal{M}$ being smooth, and for the modified set of admissible deformations

$$
\mathcal{A}_{a}:=\left\{\phi \in \mathcal{A} \mid \operatorname{det} g^{-1}[\phi]=1\right\}
$$

there exists a minimizing deformation $\phi$ in $\mathcal{A}_{a}$. If in addition we assume that $p>2$ and require that admissible deformations $\phi$ fulfill the constraint

$$
\operatorname{area}(\Omega) \leq \operatorname{area}\left(x^{-1}[\phi](\Omega)\right)
$$

then the minimizer $\phi$ is continuous and there exists a continuous deformation $\psi$ which is almost everywhere the inverse of $\phi$.

Proof We can reformulate the additional constraint requiring that $\operatorname{det} D \phi=\sqrt{\operatorname{det} g}$. But det $D \phi$ is weakly continuous on $\mathcal{A}$. Hence $\mathcal{A}_{a}$ is weakly closed. Thus, we can proceed as in the proof of Theorem 4.2. We only have to ensure that there is at least one area preserving, homeomorphic parameterization $x\left[\phi^{0}\right]$ for some deformation $\phi^{0}$. But the existence of such a parameterization has been established by Moser and Dacorogna $[13,14]$.

In the same spirit we have a corresponding result on conformal parameterizations:

Corollary 4.5 (Existence of an optimal, conformal parameterization) Under the assumptions of Theorem 4.2 with $\Omega$ being of disc type, and for

$$
\mathcal{A}_{c}:=\{\phi \in \mathcal{A} \mid \gamma=\Gamma\}
$$

with $p>2$, there exists a minimizing deformation $\phi$ in $\mathcal{A}_{c}$. Thus, there is an optimal parameterization $x[\phi]$ in the class of conformal maps.

Before proving the corollary above let us discuss some basic facts from complex analysis. Assume that a conformal map $\phi: B_{1}(0) \rightarrow \mathbb{R}^{2}$ on the unit disc $B_{1}(0)$ with non-vanishing determinant is given. We denote the points of $B_{1}(0)$ by $z=(x, y)$, and the components of $\phi$ by $\phi=(u, v)$. Conformality means that the system

$$
\left\|\phi_{x}\right\|=\left\|\phi_{y}\right\|, \quad \phi_{x} \cdot \phi_{y}=0
$$

is solved by $\phi$. Due to the fact that $\operatorname{det}\left(\phi_{x}, \phi_{y}\right) \neq 0$, we can conclude that there is a rotation $R_{\pi / 2}$ by $\pi / 2$ such that $\phi_{x}= \pm R_{\pi / 2} \phi_{y}$ and in the case of orientation 
preserving maps $\phi$ the Cauchy-Riemann equations $u_{x}=v_{y}, u_{y}=-v_{x}$ are valid. Thus, a conformal, orientation preserving mapping is holomorphic. On the other hand, it is obvious that the Cauchy-Riemann equations imply Eq. (9).

Proof [Corollary 4.5] It is well known that there is one conformal, orientation preserving parameterization $x: B_{1}(0) \rightarrow \mathbb{R}^{3}$ of the surface patch $\Omega$ over the unit disc $B_{1}(0)$. Let us consider a minimizing sequence of conformal maps $\psi^{k}: \Omega \rightarrow \mathbb{R}^{3}$. By the above considerations, these parameterizations induce a sequence of biholomorphic maps $\phi^{k}: B_{1}(0) \rightarrow \omega^{k}$ with $\phi^{k}:=\psi^{k} \circ x$ and $\omega^{k}:=\psi^{k}(\Omega)$. This sequence is bounded as in Theorem 4.2 on account of a generalized Poincaré inequality and the embedding $H^{1, p} \hookrightarrow C^{0}$. By Montel's theorem, a uniformly bounded sequence of holomorphic functions is normal [31, Chapter 14]. Thus, we can find a subsequence converging uniformly to a holomorphic map $\phi: B_{1}(0) \rightarrow \omega$. Finally, $\psi^{k}:=\phi^{k} \circ x^{-1}$ converges uniformly to the inverse $\psi=\phi \circ x^{-1}$ of a conformal parameterization.

\section{Discussion}

Our parameterization approach is characterized by

- its close relation to non-linear elasticity, a classical and well-developed field in mechanics;

- a rigorous mathematical foundation leading to one geometrically meaningful class of parameterization energies (Theorem 3.1 shows that our energy already reflects the most general geometrically meaningful case);

- a continuous model independent of the actual triangle grid allowing a straightforward discretization (see Section 7 below);

- existence results for optimal parameterizations, including those with the additional restriction of either conformality or area preservation; and

- a flexible set of parameters offered to the user to formulate preferences for the parameterization, in particular when $\alpha_{a}$ dominates one obtains a nearly area preserving parameterization (see Fig. 3), whereas a large value for $\alpha_{c}$ leads to almost conformal parameterizations.

Additionally we make the following observations.

For $\alpha_{a}=\alpha_{c}=0$ the Euler-Lagrange equations for our minimization problem turn into a system of linear equations and the parameter mapping $x[\phi]$ is a harmonic map. This derives from the fact that $\operatorname{tr}(C[\phi])$ is the squared magnitude of the first derivatives of the map $\phi \circ x^{-1}$, namely, the integrand of the Dirichlet energy. In fact, a non-zero weighted length term is essential for the well-posedness of the free boundary approach. As a consequence, methods which solely consider the control of area and angle will in general lack a proper, continuous limit problem for succes- 

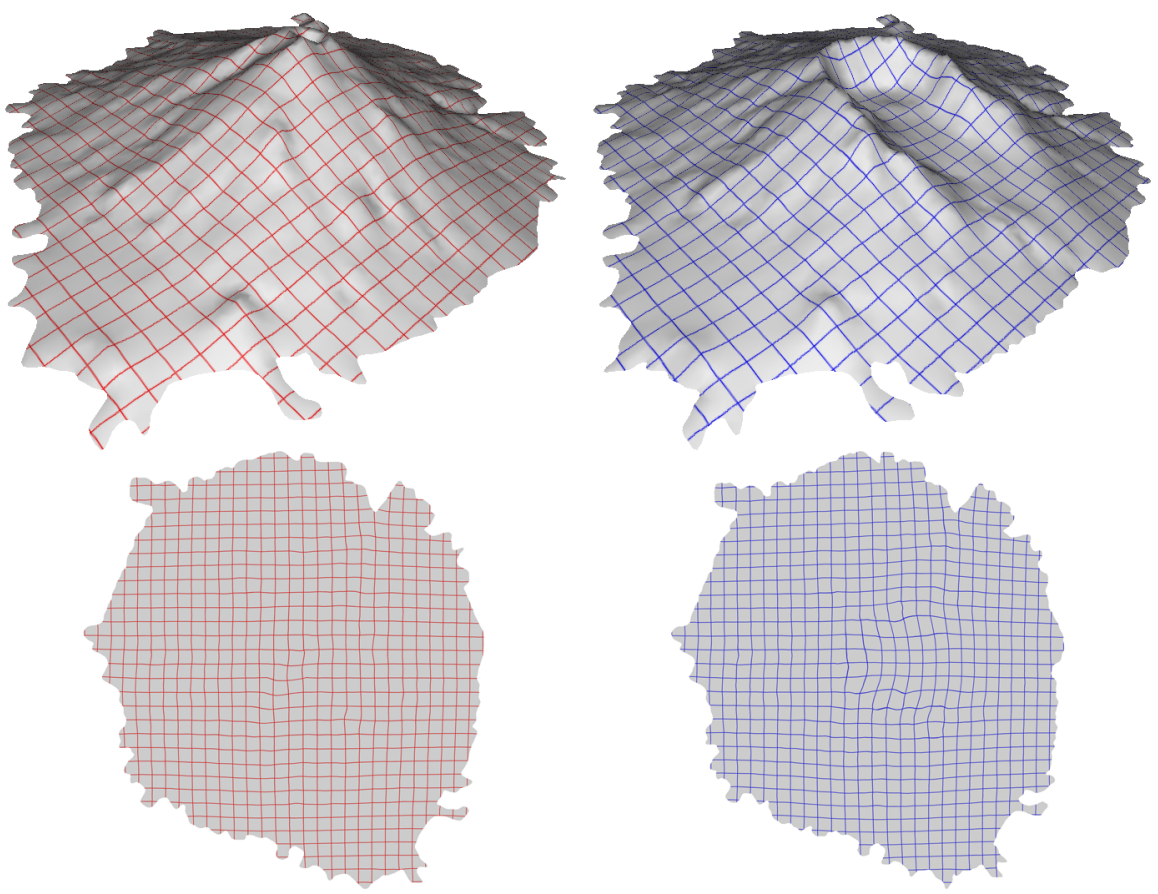

Fig. 3. Area preserving parameterizations of geographic data for Mount St. Helens, before and after its eruption in 1980 (shown here: reconstructed surface and parameter domain).

sively refined surface triangulations. Additionally, Moser [13] showed that control of area — directly or indirectly via a conformal energy — is similarly critical in that it ensures the local injectivity on the discrete level of triangulations.

Generally speaking, there is no single set of optimal parameters to match the user's requirements. Instead, based on their clear geometric meaning, the parameters can be tuned to certain applications. The linear terms $\alpha_{l}, \alpha_{a}, \alpha_{c}$ control the relative importance of length, area and angle distortion in the parameterization, respectively, whereas the exponents $p, r, s, t$ act to prevent high distortion as measured by their related energies. Lemma 3.2 gives a formula for the dependent parameter $\beta$.

Uniqueness of optimal parameterizations cannot be expected in general. Classical non-uniqueness examples in elasticity [16] give a recipe for a corresponding construction in the case of parameterizations. Furthermore, a variational energy which ensures injectivity is generically non-linear (see the injectivity discussion in [16]). Hence, linear methods $[3,4,32]$ face difficulties for parameterizations which have large distortion. These issues are well known as the presence of negative cotangent weights for discrete harmonic maps and the convexity conditions for Floater's positive weights.

Finally, we observe that the various measures of the singular values of the Jacobian of the parameter mapping used in earlier approaches are closely related to our measures involving $\Gamma$ and $\gamma$. For example, the $l_{2}$ norm of the singular values of the Jacobian used by Sander et al. is essentially our length control energy. Hormann 
and Greiner, in their use of the condition number of the Jacobian, use the same local quantity, $a^{2} / d$, to penalize non-conformality as we do. A similar observation holds for the energy terms used by Desbrun et al. and Lévy et al.

\section{Euler-Lagrange Equations}

In the general case of an energy

$$
E[\phi]=\int_{\omega} \hat{W}(D \phi) \sqrt{\operatorname{det} g} \mathrm{~d} \xi
$$

with the energy density $\hat{W}: \mathbb{R}^{2,2} \rightarrow \mathbb{R} ; B \mapsto f(B)$ we obtain

$$
\begin{aligned}
E^{\prime}[\phi](\vartheta) & =\int_{\omega} \partial_{A} \hat{W}(D \phi)(D \vartheta) \sqrt{\operatorname{det} g} \mathrm{~d} \xi \\
E^{\prime \prime}[\phi](\vartheta, \zeta) & =\int_{\omega} \partial_{A}^{2} \hat{W}(D \phi)(D \vartheta, D \zeta) \sqrt{\operatorname{det} g} \mathrm{~d} \xi .
\end{aligned}
$$

Explicit formulas for the energy density defined in Eq. (6) are given in the Appendix. The representation of the first and the second derivative in the $L^{2}$ scalar product on the space of deformations $H^{1,2}\left(\omega, \mathbb{R}^{2}\right)$ define the gradient $\nabla E$ and the Hessian $\nabla^{2} E$, respectively:

$$
\begin{aligned}
(\nabla E[\phi], \vartheta) & :=E^{\prime}[\phi](\vartheta), \\
\left(\nabla^{2} E[\phi] \vartheta, \psi\right) & :=E^{\prime \prime}[\phi](\vartheta, \psi) .
\end{aligned}
$$

Then the necessary condition for a sufficiently smooth deformation $\phi$ to minimize the energy $E$ is $\nabla E[\phi]=0$. We have already seen this for a sufficiently smooth deformation $\phi$ which leads to an isometric parameterization $x[\phi]$ and under the assumption of Lemma 3.2 we obtain that $\nabla^{2} E[\phi]$ is positive definite. With respect to the later discretization, the gradient and Hessian of the energy are required to set up a Newton method. Due to the required normalization with respect to rigid body motions, we actually have to solve a saddle point problem. In fact, we replace the energy $E$ by an energy $E_{c}: \mathcal{A} \times \mathbb{R}^{3} \rightarrow \mathbb{R} ;(\phi, \lambda) \mapsto E_{c}[\phi, \lambda]$ with

$$
E_{c}[\phi, \lambda]=E[\phi]+\int_{\omega} \lambda_{1} \phi(\xi)_{1}+\lambda_{2} \phi(\xi)_{2}+\lambda_{3}\left(\phi(\xi)_{2} \xi_{1}-\phi(\xi)_{1} \xi_{2}\right) \mathrm{d} \xi
$$

Instead of solving $\nabla_{\phi} E[\phi]=0$ for $\phi$, one now solves the equation

$$
\nabla_{(\phi, \lambda)} E_{c}[\phi, \lambda]=0
$$


for the deformation $\phi$ and the Lagrange multipliers $\lambda_{1}, \lambda_{2}, \lambda_{3}$.

\section{Discretization}

Suppose $\Omega_{h}$ is a triangular mesh on some discrete surface $\mathcal{M}_{h}$ and $\omega_{h}$ is the corresponding admissible triangular mesh in the parameter space $\mathbb{R}^{2}$ of an initial piecewise affine and continuous parameterization $x$. Let $T$ denote the triangles of $\omega_{h}$ and suppose that $\bigcup_{T \in \omega_{h}}=\omega$. Let $V_{h}$ be the space of piecewise affine, continuous functions on $\omega_{h}$. We denote discrete functions and in particular discrete deformations by upper case letters. Furthermore, we suppose $\left\{\Phi^{k}\right\}_{k=1 \cdots n}$ to be the nodal basis of $V_{h}$ for an index set $K$ corresponding to the set of nodes $\xi^{k}$ of $\omega_{h}$. Hence, $\left\{\Phi^{i k}\right\}_{i=1,2 ; k=1, \cdots n}$ with $\Phi^{i k}:=\Phi^{k} e^{i}$ forms a basis of the discrete space of deformations $V_{h}^{2}$. Given a discrete deformation $\Phi \in V_{h}^{2}$ the coordinate vector will be denoted by $\bar{\Phi}$, where $\bar{\Phi}_{i k}=\Phi\left(\xi^{k}\right)_{i}$. On each triangle $T$ of $\omega_{h}$, the derivative $D \Phi^{i k}$ is a constant matrix in $\mathbb{R}^{2,2}$. Also, the discrete metric $G=\left(G_{i j}\right)_{i j} \in \mathbb{R}^{2,2}$ with $G_{i j}:=\partial_{i} X \cdot \partial_{j} X$ is constant on $T$. For a discrete deformation $\Phi$, we obtain

$$
E[\Phi]=\sum_{T \in \omega_{h}} W\left(\iota_{A(T) G(T)^{-1} A(T)^{T}}\right) \sqrt{\operatorname{det} G(T)}|T|
$$

where on each triangle $T$ we define the constant matrices $A(T):=\left.D \Phi\right|_{T}$ and $G(T):=\left.G\right|_{T}$. Let $\bar{E}: \mathbb{R}^{2 n} \rightarrow \mathbb{R}$ be the discrete counterpart of the energy functional $E$ defined on coefficient vectors with respect to the basis $\left\{\Phi^{i k}\right\}$, i.e.,

$$
\bar{E}[\bar{\Phi}]:=E[\phi]
$$

Then, $\nabla \bar{E} \in \mathbb{R}^{2 n}$ with

$$
(\nabla \bar{E}[\bar{\Phi}])_{i k}=\left(\nabla E[\Phi], \Phi^{i k}\right)=E^{\prime}[\Phi]\left(\Phi^{i k}\right)
$$

and $\nabla^{2} \bar{E} \in \mathbb{R}^{2 n, 2 n}$ with

$$
\left(\nabla^{2} \bar{E}[\bar{\Phi}]\right)_{i k j l}=\left(\nabla^{2} E[\Phi] \Phi^{i k}, \Phi^{j l}\right)=E^{\prime \prime}[\Phi]\left(\Phi^{i k}, \Phi^{j l}\right)
$$

Similarly, we define $\bar{E}_{c}[\bar{\Phi}, \lambda]$ corresponding to the minimization problem with moment constraints. Then the Hessian of $\bar{E}_{c}$ reads as follows:

$$
\nabla^{2} \bar{E}_{c}[\bar{\Phi}, \lambda]=\left(\begin{array}{cc}
\nabla^{2} \bar{E}[\bar{\Phi}] & M \\
M^{T} & 0
\end{array}\right), \quad M=\left(\begin{array}{ccc}
\mathbf{m} & 0 & -\mathbf{m} \\
0 & \mathbf{m} & \mathbf{m}
\end{array}\right),
$$




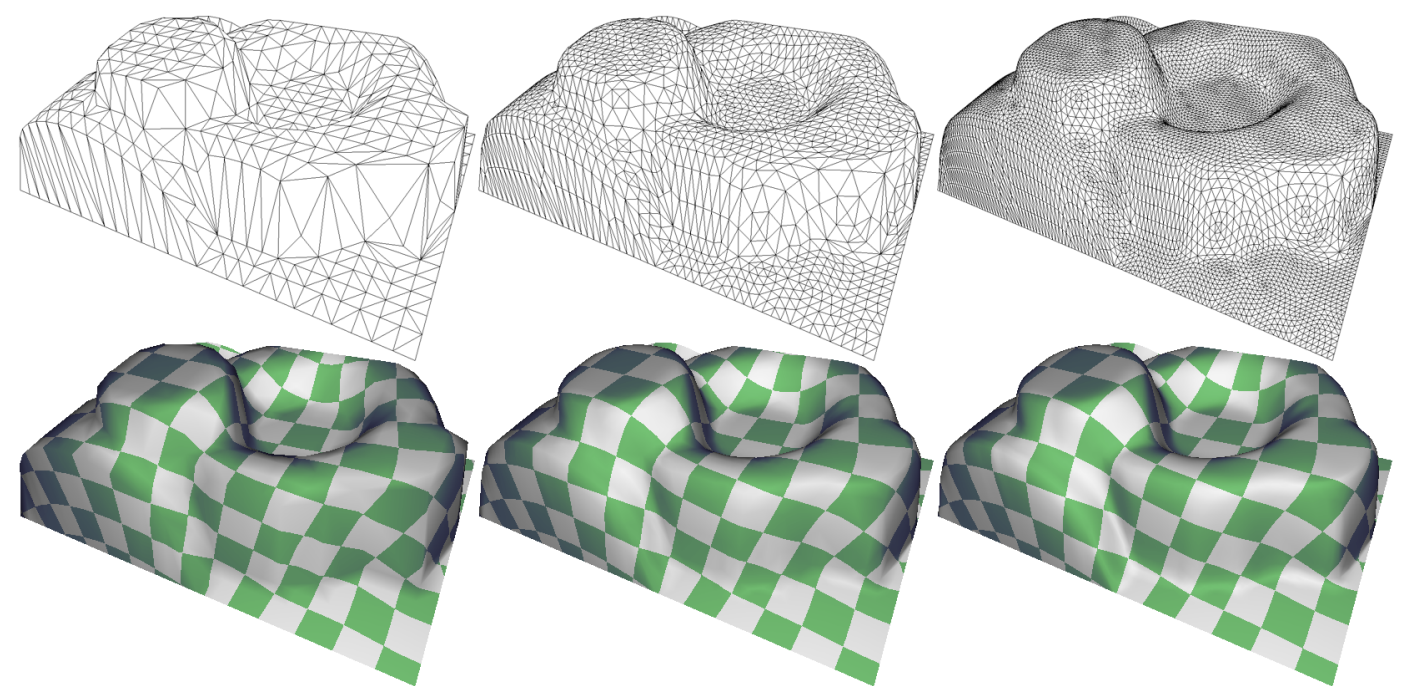

Fig. 4. Successive refinement of a triangulation demonstrates the convergence of the parameterization, due to the fact that the different discretizations minimize consistent approximations of the same continuous energy functional.

where $\mathbf{m}:=\left(\int_{\omega_{h}} \Phi^{k}\right)_{k}$ is the vector of the masses of the basis functions $\Phi^{k}$. Finally, we have collected all the ingredients of a fully discrete Newton method, which we list here in pseudo code:

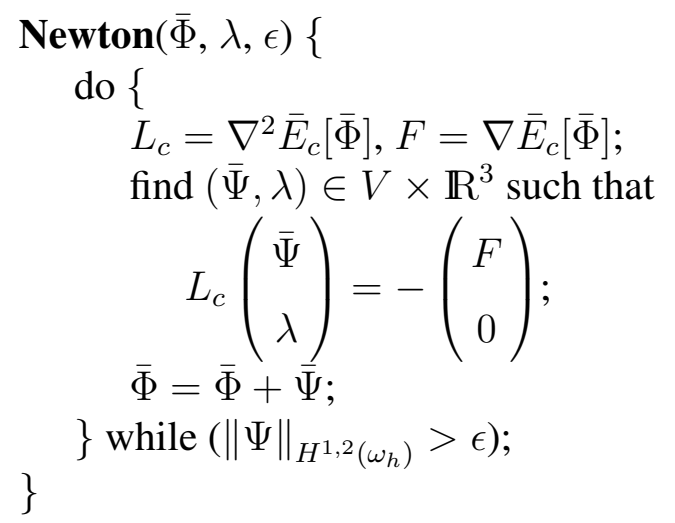

We refer to the Appendix for explicit formulas for the derivatives of $\hat{W}$ defined in Eq. (6). For the solution of the linear saddle point problem, one could apply Uzawa's algorithm [33]. We simply use a preconditioned standard conjugate gradient algorithm. We have never observed any difficulty in practice even though theoretically the matrix $L_{c}$ is not positive definite. As a preconditioner, we apply a diagonal preconditioning to $\nabla^{2} \bar{E}$. 


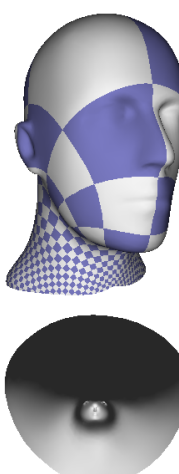

$[3,4]$

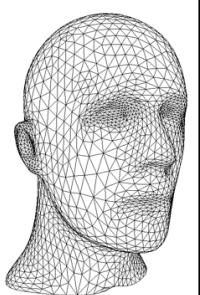

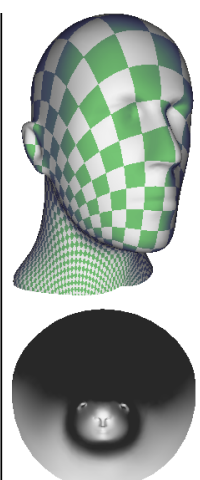
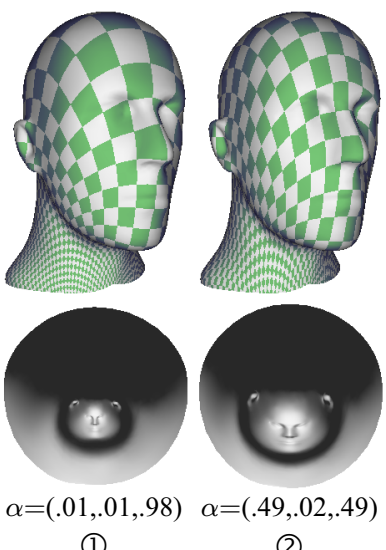

(1)

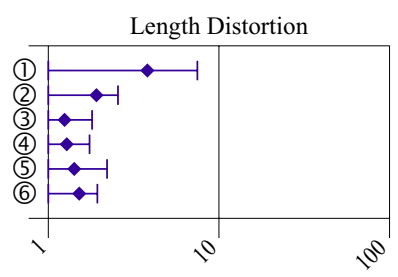

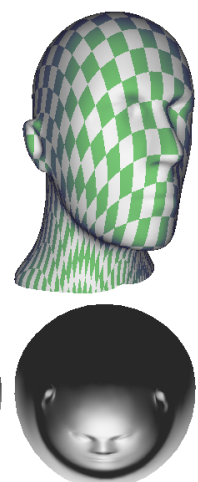
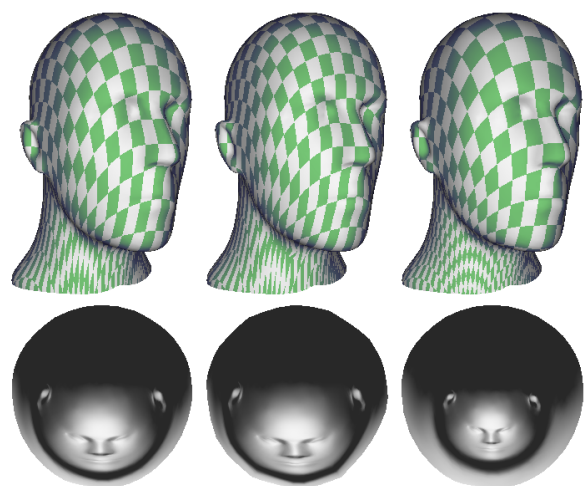

(3)

(4)

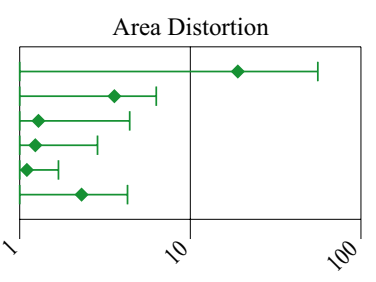

(6)

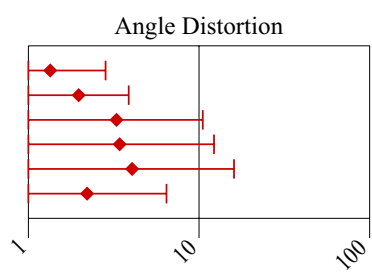

Fig. 5. The parameterization is controlled by parameters $\alpha=\left(\alpha_{l}, \alpha_{a}, \alpha_{c}\right)$ which capture the trade-off between length, area and angle preservation, respectively. Texture maps of the initial parameterization on the left and solutions for different parameter settings are shown on top, with the parameter domain beneath. A plot of the maximum, minimum and mean distortion is shown below. Notice that the measured distortion tends to to reflect the trade-offs in the choice of parameters.

\section{Results}

Our parameterization method is implemented by an algorithm which finds the optimal parameterization for a given surface, starting from an initial parameterization which is also provided. The final parameterization is optimal in the sense that it minimizes the energy in Eq. (6), controlled by the parameters $\alpha=\left(\alpha_{l}, \alpha_{a}, \alpha_{c}\right)$ which balance the trade-off between length, area and angle preservation, respectively. For the sake of simplicity, we restrict ourselves to the exponents $p=2$, $q=2, r=2$, and $t=1$. The remaining parameters must satisfy the conditions in Lemma 3.2, and furthermore we require that $\alpha_{l}+\alpha_{a}+\alpha_{c}=1$. Fig. 5 demonstrates our algorithm for a variety of parameter values. Given the initial parameterization on the left, each parameterization on the right is obtained by minimizing the corresponding energy with the Newton method above.

The relative importance of the three competing parameters $\alpha_{l}, \alpha_{a}, \alpha_{c}$ is reflected in the distortion of the final parameterization. Recall that distortion can be measured as a function of the maximum and minimum eigenvalues $\Gamma, \gamma$ of the first fundamental form of the parameterization function. We calculate the mean distortion for a parameterization as the geometric sum of the contributions from each triangle in 

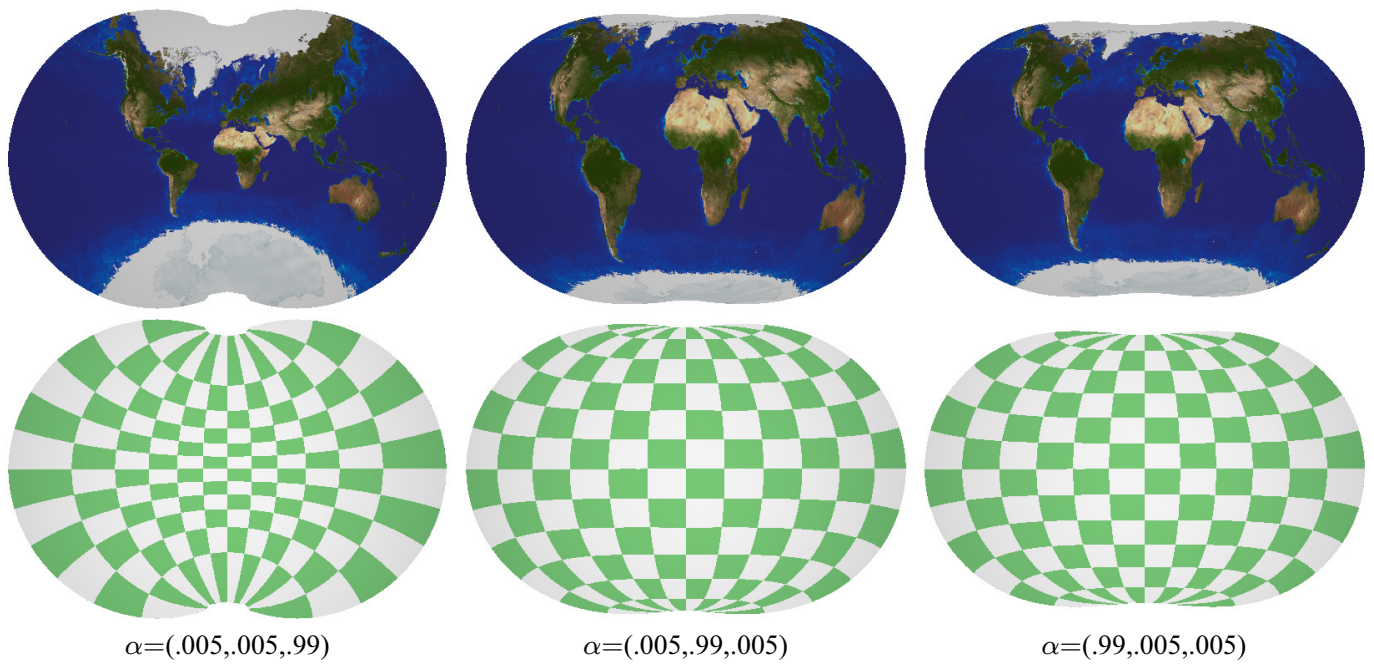

Fig. 6. Flattening the earth. The sphere is opened with a semi-circular cut and its parameterization is optimized for angle, area and length preservation, respectively. Here texture maps show the deformation of the surface in the parameter domain.

our discretization. Popular measures of distortion for angles $(\sqrt{\Gamma / \gamma})$, area $(\sqrt{\Gamma \gamma})$ and length $(\sqrt{\Gamma+\gamma})$ for Fig. 5 are reported in the bottom graphs, where the extreme deformations in the parameterization functions clearly show the trade-offs between the different distortion measures. This correspondence is further explored in Fig. 6, which demonstrates the effect of minimizing the different energy terms on parameterizations of a sphere that has been cut.

All of our results were computed using a simple Newton method to perform the energy minimization, starting from a reasonable initial guess. In our implementation, we use an adaptive line search [34] to ensure a robust minimization. At each step, we compute a search direction $\bar{\Psi}$ by solving the sparse linear system described in Section 7. The number of Newton iterations needed to minimize the energy depends on the initial parameterization. If the optimal parameterization is quite different, performance can be adversely affected. Furthermore, local injectivity is guaranteed in the discrete method through step size control. Specifically, the condition for sufficient decrease of the energy is violated in the presence of a fold, due to the fact that the area energy functional grows unbounded. Therefore, folds cannot appear from an admissible deformation.

The initial parameterization plays an important role, both in preventing overfolding in the final solution, and in improving performance. We have found that the natural conformal map [3,4] works very well in practice: it is cheap to compute, it has natural boundary conditions and it has low angle distortion. We use this initial parameterization in all of our examples. Finally, we can improve the performance and the stability of our method by incrementally varying $\alpha$ to reach the desired parameterization. This tends to reduce the number of Newton iterations, because the starting parameterization at each increment is very close to optimal. 


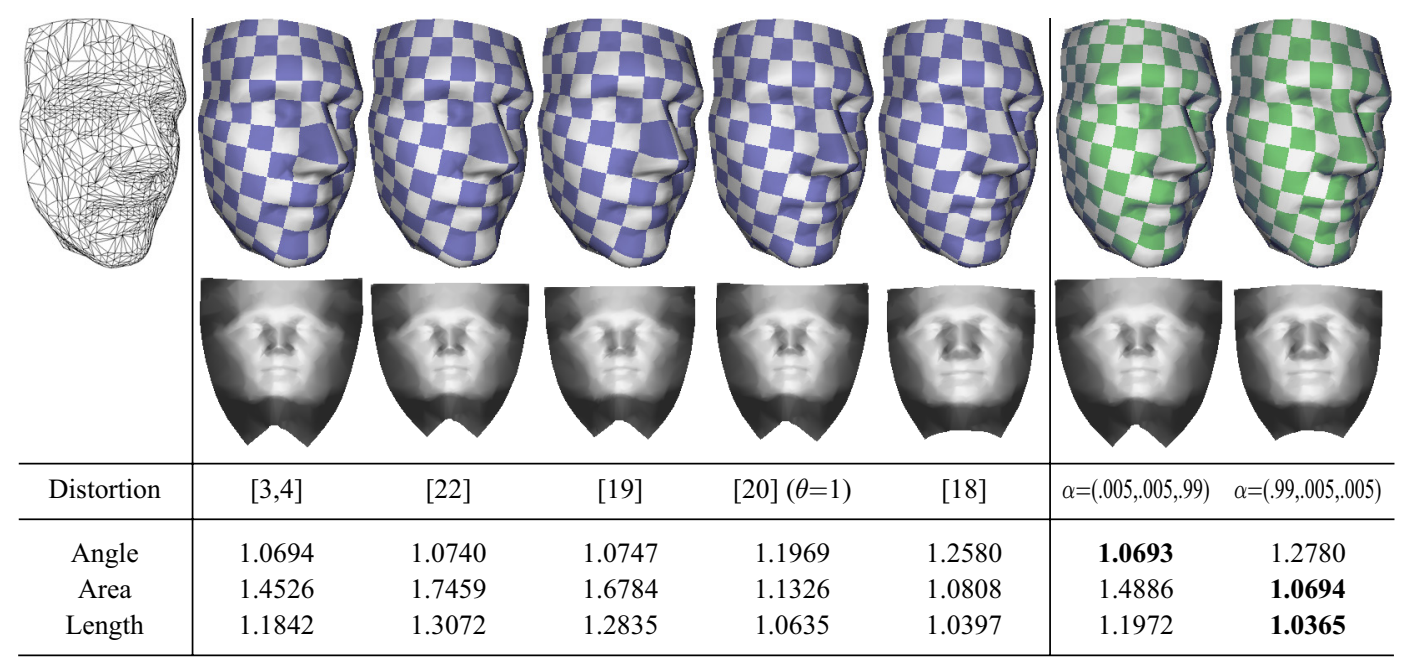

Fig. 7. A comparison of previous work to parameterizations generated by our method. Unlike other methods, we are not restricted to optimizing for only one notion of distortion, as demonstrated in the table of mean distortion measurements above.

The continuous energy model that we use has several distinct advantages. The previous examples demonstrate the unlimited range of parameterizations that can be achieved by varying $\alpha$. Additionally, since our energy is defined in the continuous setting, the solution to the minimization problem is insensitive to the particular discretization of the surface. This fact can be observed because different discretizations minimize consistent approximations of the same continuous energy functional. In Fig. 4, we consider the successive refinement of a triangular grid that represents a stamped mechanical part. Notice how the parameterization converges rapidly, and its main features are apparent even at the coarsest resolution. Not surprisingly, the number of Newton steps taken for each of these examples is also identical.

The results shown here were obtained in a matter of seconds to a few minutes on a $2 \mathrm{GHz}$ Intel Pentium processor. The execution time depends on the number of Newton iterations, which in turn depends on the quality of the initial parameterization. For the example in Fig. 1, a hierarchical solver was used to solve the linear system for each Newton step, which drastically reduced the time to generate the parameterization. Due to the straightforward finite element discretization of the previous section, numerous classical numerical methods apply, however their discussion is beyond the scope of this paper.

\section{Conclusions}

We have presented a parameterization method that gives the user flexible control over the trade-off between area, length and angle distortion. Fig. 5 demonstrates this 
control with examples that range from angle preserving to area preserving, and a comprehensive balance with length distortion in the rest. This contrasts our method from previous work, which largely focused on optimizing for only one measure of distortion, or possibly using linear combinations of energies. Fig. 7 demonstrates that this trade-off is highly effective. For example, when emphasizing conformality, we achieve a smaller mean distortion than methods which were geared towards the construction of conformal mappings. The same is true for the stretch minimization (note however that we only minimize the $l_{2}$ error with respect to length, not the $l_{\infty}$ error as Sander et al. do). Because of the inherent non-linearity of our method, we find that the discrete conformal mappings which result from linear systems provide us with an excellent starting point for our optimization.

The fundamental advantages of our approach stem from its foundation in the classical theory of rational mechanics. This allows us to rephrase the surface parameterization problem as one of optimizing a unified energy from a small class of admissible energy densities that simultaneously captures angle, area and length distortion. Remarkably, this uniqueness follows directly from the axioms of isotropy and frame invariance. Furthermore, putting this method into practice is simple and well understood from the point of robust numerical methods.

In some settings it may be desirable to explicitly introduce anisotropy. Here too elasticity theory can provide tools whose exploration should be pursued in future work.

Acknowledgements This work was supported in part by the Deutsche Forschungsgemeinschaft, NSF (DMS-0220905, DMS-0138458, ACI-0219979), the DOE (W7405-ENG-48/B341492), nVidia, and Alias|Wavefront. Special thanks is due to Peter Schröder for his many insightful comments. We also wish to thank the authors cited in Fig. 7 for generously providing us with parameterization data. The St. Helens dataset appears courtesy of Kai Hormann.

\section{References}

[1] M. S. Floater, K. Hormann, Surface parameterization: a tutorial and survey, in: Multiresolution in Geometric Modelling, Springer, 2004.

[2] M. Eck, T. D. DeRose, T. Duchamp, H. Hoppe, M. Lounsbery, W. Stuetzle, Multiresolution analysis of arbitrary meshes, in: Proceedings of SIGGRAPH, 1995, pp. 173-182.

[3] M. Desbrun, M. Meyer, P. Alliez, Intrinsic parameterizations of surface meshes, in: Eurographics 2002, 2003, pp. 209-218.

[4] B. Lévy, S. Petitjean, N. Ray, J. Maillot, Least squares conformal maps for automatic texture atlas generation, ACM Transactions on Graphics 21 (3) (2002) 362-371. 
[5] X. Gu, S.-T. Yau, Computing conformal structures of surfaces, Communications in Informations and Systems (2002) 121-146.

[6] L. Lichtenstein, Zur Theorie der konformen Abbildung. Konforme Abbildungen nichtanalytischer singularitätenfreier Flächenstücke auf ebene Gebiete, Bull. Acad. Sci. Cracovie, Cl. Sci. Math. Nat. A (1916) 192-217.

[7] C. Morrey, Multiple Integrals in the Calculus of Variations, Springer-Verlag, NewYork, 1966.

[8] J. Jost, Two-dimensional geometric variational problems, Wiley-Interscience, 1991.

[9] F. Sauvigny, Curvature estimates for immersions of minimal surface type via uniformization and theorems of Bernstein type, Manuscr. Math. 67 (1) (1990) 69-97.

[10] S. Hildebrandt, H. von der Mosel, On globally conformal mappings, Preprint, SFB 611, Universität Bonn .

[11] U. Dierkes, S. Hildebrandt, A. Küster, O. Wohlrab, Minimal Surfaces, SpringerVerlag, 1992.

[12] S. Angenent, S. Haker, A. Tannenbaum, Minimizing flows for the Monge-Kantorovich problem, SIAM J. Math. Anal. 35 (1) (2003) 61-97.

[13] J. Moser, On the volume elements on a manifold, Trans. Amer. Math. Soc. 120 (1965) 286-294.

[14] B. Dacorogna, J. Moser, On a partial differential equation involving the Jacobian determinant, Ann. Inst. H. Poincaré Anal. Non Linéaire 7 (1) (1990) 1-26.

[15] J. E. Marsden, T. J. R. Hughes, Mathematical Foundations of Elasticity, Prentice-Hall, Englewood Cliffs, 1983.

[16] P. G. Ciarlet, Three-Dimensional Elasticity, Elsevier, New York, 1988.

[17] R. W. Ogden, Non-Linear Elastic Deformations, John Wiley, 1984.

[18] P. V. Sander, J. Snyder, S. J. Gortler, H. Hoppe, Texture mapping progressive meshes, Proceedings of SIGGRAPH 2001 (2001) 409-416.

[19] K. Hormann, G. Greiner, MIPS: An efficient global parametrization method, in: Curve and Surface Design: Saint-Malo 1999, 2000, pp. 153-162.

[20] P. Degener, J. Meseth, R. Klein, An adaptable surface parameterization method, in: Proc. 12th Int. Meshing Roundtable, 2003, pp. 201-213.

[21] O. Sorkine, D. Cohen-Or, R. Goldenthal, D. Lischinski, Bounded-distortion piecewise mesh parameterization, in: IEEE Visualization, 2002, pp. 355-362.

[22] A. Sheffer, E. de Sturler, Parameterization of faceted surfaces for meshing using angle based flattening, Engineering with Computers 17 (3) (2001) 326-337.

[23] B. Aksoylu, A. Khodakovsky, P. Schröder, Multilevel solvers for unstructured surface meshes, To appear, SIAM J. Sci. Comp . 
[24] B. Dacorogna, Direct Methods in the Calculus of Variations, Appl. Math. Sciences 78, Springer-Verlag, Berlin, 1989.

[25] L. Ambrosio, N. Fusco, D. Pallara, Functions of bounded variation and free discontinuity problems, Oxford University Press, 2000.

[26] J. Ball, Global invertibility of Sobolev functions and the interpenetration of matter, Proc. Roy. Soc. Edinburgh 88A (1988) 315-328.

[27] V. Šverák, Regularity properties of deformations with finite energy, Arch. Rat. Mech. Anal. 100 (1988) 105-127.

[28] C. Morrey, Quasi-convexity and lower semicontinuity of multiple integrals, Pac. J. Math. 2 (1952) 25-53.

[29] J. Ball, Convexity conditions and existence theorems in nonlinear elasiticity, Arch. Rat. Mech. Anal. 63 (1977) 337-403.

[30] P. G. Ciarlet, J. Necas, Injectivity and self-contact in nonlinear elasticity, Arch. Rational Mech. Anal. 97.

[31] W. Rudin, Real and complex analysis, McGraw-Hill, 1987.

[32] M. S. Floater, Mean value coordinates, Computer Aided Geometric Design 20 (1) (2003) 19-27.

[33] X. Cheng, On the nonlinear inexact Uzawa algorithm for saddle-point problems, SIAM J. Numer. Anal. 37 (6) (2000) 1930-1934.

[34] J. Bonnans, J. C. Gilbert, C. Lemarechal, C. Sagastizabal, Numerical Optimization, Springer, 2003.

\section{Appendix}

Here we provide the concrete formulas for the derivatives of the energy density $\hat{W}(A)=W\left(\iota_{A g^{-1} A^{T}}\right)$ defined in Eq. (6). By straightforward computation one obtains for the derivatives of $W$ appearing in the fully discrete $\nabla \bar{E}$ in Eq. (10) and $\nabla^{2} \bar{E}$ in Eq. (11):

$$
\begin{aligned}
(\nabla \bar{E})_{i k} & =\sum_{T \in \omega_{h}}|T| \partial_{A} \hat{W}(A(T)) B^{i k}(T) \sqrt{\operatorname{det} G(T)}, \\
\left(\nabla^{2} \bar{E}\right)_{i k j l} & =\sum_{T \in \omega_{h}}|T| \partial_{A}^{2} \hat{W}(A(T)) B^{i k}(T) B^{j l}(T) \sqrt{\operatorname{det} G(T)},
\end{aligned}
$$

where $A(T)=\left.D \Phi\right|_{T}$ as above and $B^{r s}(T)=\left.D \Phi^{r s}\right|_{T}$. Furthermore, 


$$
\begin{aligned}
& \partial_{A} \hat{W}(A)(C)=\alpha_{l} \frac{p}{2} a^{\frac{p}{2}-1} \frac{\partial a}{\partial A}(C)+\alpha_{a}\left(\frac{r}{2} d^{\frac{r}{2}-1}-\beta \frac{s}{2} d^{-\frac{s}{2}-1}\right) \frac{\partial d}{\partial A}(C) \\
& +\alpha_{c} t\left(\frac{a^{2}}{d}-4\right)^{t-1}\left(\frac{2 a}{d} \frac{\partial a}{\partial A}(C)-\frac{a^{2}}{d^{2}} \frac{\partial d}{\partial A}(C)\right), \\
& \partial_{A}^{2} \hat{W}(A)(C, H)=\alpha_{l} \frac{p}{2}\left(\frac{p}{2}-1\right) a^{\frac{p}{2}-2} \frac{\partial a}{\partial A}(C) \frac{\partial a}{\partial A}(H)+\alpha_{a} \frac{p}{2} a^{\frac{p}{2}-1} \frac{\partial^{2} a}{\partial A^{2}}(C, H) \\
& \alpha_{a}\left(\frac{r}{2}\left(\frac{r}{2}-1\right) d^{\frac{r}{2}-2}+\beta \frac{s}{2}\left(\frac{s}{2}+1\right) d^{-\frac{s}{2}-2}\right) \frac{\partial d}{\partial A}(C) \frac{\partial d}{\partial A}(H) \\
& +\alpha_{a}\left(\frac{r}{2} d^{\frac{r}{2}-1}-\beta \frac{s}{2} d^{-\frac{s}{2}-1}\right) \frac{\partial^{2} d}{\partial A^{2}}(C, H) \\
& +\alpha_{c} t\left(\frac{a^{2}}{d}-4\right)^{t-1}\left(\frac{2 a}{d} \frac{\partial^{2} a}{\partial A^{2}}(C, H)+\frac{2}{d} \frac{\partial a}{\partial A}(C) \frac{\partial a}{\partial A}(H)\right. \\
& -\frac{2 a}{d^{2}} \frac{\partial a}{\partial A}(C) \frac{\partial d}{\partial A}(H)-\frac{a^{2}}{d^{2}} \frac{\partial^{2} d}{\partial A^{2}}(C, H) \\
& \left.-\frac{2 a}{d^{2}} \frac{\partial d}{\partial A}(C) \frac{\partial a}{\partial A}(H)+\frac{2 a^{2}}{d^{3}} \frac{\partial d}{\partial A}(C) \frac{\partial d}{\partial A}(H)\right) \\
& +\alpha_{c} t(t-1)\left(\frac{a^{2}}{d}-4\right)^{t-2}\left(\frac{2 a}{d} \frac{\partial a}{\partial A}(C)-\frac{a^{2}}{d^{2}} \frac{\partial d}{\partial A}(C)\right) \\
& \left(\frac{2 a}{d} \frac{\partial a}{\partial A}(H)-\frac{a^{2}}{d^{2}} \frac{\partial d}{\partial A}(H)\right)
\end{aligned}
$$

and using the fact that $\partial_{A}\left(A^{-1}\right)(C)=-A^{-1} C A^{-1}$,

$$
\begin{aligned}
\frac{\partial a}{\partial A}(C) & =2 \operatorname{tr}\left(A g^{-1} C^{T}\right), \\
\frac{\partial^{2} a}{\partial A^{2}}(C, H) & =2 \operatorname{tr}\left(H g^{-1} C^{T}\right), \\
\frac{\partial d}{\partial A}(C) & =2(\operatorname{det} A)^{2}(\operatorname{det} g)^{-1} \operatorname{tr}\left(A^{-1} C\right), \\
\frac{\partial^{2} d}{\partial A^{2}}(C, H) & =2(\operatorname{det} A)^{2}(\operatorname{det} g)^{-1}\left(2 \operatorname{tr}\left(A^{-1} C\right) \operatorname{tr}\left(A^{-1} H\right)-\operatorname{tr}\left(A^{-1} H A^{-1} C\right)\right) .
\end{aligned}
$$

